\title{
Les contraintes entomologiques de la culture du niébé et leur mode de gestion par les producteurs dans les régions de Maradi et Zinder au Niger
}

\author{
Ousséina ABDOULAYE ZAKARI ${ }^{1,2}$, Ibrahim BAOUA ${ }^{2 *}$, Laouali AMADOU ${ }^{1}$, \\ Manuele TAMÒ ${ }^{3}$ et Barry Robert PITTENDRIGH ${ }^{4}$ \\ ${ }^{1}$ Institut National de la Recherche Agronomique du Niger (INRAN) BP 240 Maradi, Niger. \\ ${ }^{2}$ Université Dan Dicko Dankoulodo de Maradi, BP 465 Maradi, Niger. \\ ${ }^{3}$ International Institute of Tropical Agriculture (IITA), 08 BP 0932, Tri Postal, Cotonou, Bénin. \\ ${ }^{4}$ Department of Entomology, Michigan State University, East Lansing, MI 48824 USA. \\ *Corresponding author; E-mail: baoua.ibrahim@gmail.com
}

\section{REMERCIEMENTS}

L'étude a été réalisée grâce au soutien financier et matériel du Programme de Productivité Agricole en Afrique de l'Ouest (PPAAO) dans le cadre du projet "Promotion des biopesticides pour la gestion intégrée des insectes ravageurs du niébé en champ au Niger et au Burkina Faso", et du projet Legume Lab Innovation de l'Université Illinois financé par l'US Agency for International Development (USAID).

\section{RESUME}

Le niébé est une culture alimentaire et socioéconomique importante en Afrique de l'Ouest. Cependant, son rendement est bas en raison de la pression des bioagresseurs. Cette étude a été conduite auprès des producteurs du niébé pour recenser les principales contraintes entomologiques et les méthodes de lutte utilisées. Des focus groupes ont été organisés avec des groupes de 25 à 35 producteurs dans 20 villages des régions de Maradi et Zinder. Il ressort que le niébé occupe les 1/3 des surfaces cultivables et l'association mil-niébé est pratiquée par $47,4 \%$ des producteurs. Le puceron Aphis craccivora Koch et la punaise brune Clavigralla tomentosicollis Stal sont les principaux ravageurs évoqués par $80 \%$ des villages enquêtés. Maruca vitrata Fabricius n'est pas connu par $83 \%$ des répondants. Pour la gestion de ces ravageurs, $37,25 \%$ des producteurs ne font rien, $32,2 \%$ utilisent la lutte chimique avec les pesticides de synthèse. Le biopesticide à base des extraits de grains de neem est connu par 53\% des répondants, mais son mode de préparation est méconnu par $69,8 \%$ des répondants. Ces informations vont contribuer à l'élaboration d'un programme pour l'amélioration de la productivité du niébé et la réduction de la pauvreté rurale au Niger

(C) 2019 International Formulae Group. All rights reserved

Mots clés: Niébé, Maradi, Zinder, Aphis craccivora, Clavigralla tomentosicollis, Maruca vitrata.

\section{The entomological constraints of cowpea crop and their control method by farmers in the regions of Maradi and Zinder in Niger}

\begin{abstract}
Cowpea is an important food and socio-economic crop in West Africa. However, its yield is low because of bio aggressors attack. This study was conducted with producers to identify the main
\end{abstract}


entomological constraints and the control methods used. Focus groups were organized with groups of 25 to 35 producers in 20 villages of Maradi and Zinder regions. It is shown that cowpea occupies $1 / 3$ of cultivable areas and the millet-cowpea mixed cropping is done by $47.4 \%$ of producers. The aphid Aphis craccivora Koch and the pod sucking bug Clavigralla tomentosicollis Stal are the main pests stated by $80 \%$ of the surveyed villages. Maruca vitrata Fabricius is unknown by $83 \%$ of respondents. For the management of these pests, $37.25 \%$ of producers apply no control method, $32.2 \%$ use chemical control with synthetic pesticides. The neem seeds extracts biopesticide is known by $53 \%$ of respondents, but its method of preparation is unknown by $69.8 \%$ of respondents. This information will contribute to the development a program for the improvement of cowpea productivity and reduce rural poverty in Niger.

(C) 2019 International Formulae Group. All rights reserved

Keywords: Cowpea, Maradi, Zinder, Aphis craccivora, Clavigralla tomentosicollis, Maruca vitrata.

\section{INTRODUCTION}

Le niébé, Vigna Unguiculata L. Walpers (Fabaceae), est l'une des principales légumineuses alimentaire produite au monde et particulièrement en Afrique de l'Ouest. Cette culture joue un rôle dans la réduction de la pauvreté et à l'amélioration de la sécurité alimentaire en raison de son taux élevé en protéines et de son importance socioéconomique (Stoilova et Pereira, 2013; Mehinto et al., 2014). La production mondiale annuelle est d'environ 5,59 millions de tonnes pour des superficies cultivées de plus de 12,61 millions d'hectares (FAO, 2014). L'Afrique de l'Ouest produit à elle seule environ $83 \%$ de la production mondiale (FAOSTAT, 2016).

Le niébé est cultivé dans toute la bande agricole Sud du Niger. La production en 2018 est de 2282382 tonnes, et les grandes zones de production sont les régions de Maradi et Zinder (MA, 2018). Il occupe 48\% des superficies emblavées et représente le troisième produit agricole contributeur au PIB après le bétail et l'oignon (MA, 2012). Malgré son importance alimentaire et économique, le rendement du niébé est très bas, de l'ordre de 364-394 kg/ha dans les grandes zones de production (MA, 2018). La production de cette légumineuse est limitée par plusieurs contraintes biotiques et abiotiques, notamment les attaques des insectes ravageurs, la pauvreté des sols, l'accès limité des producteurs aux semences des variétés améliorées, la non disponibilité des insecticides et engrais à des prix abordables (Sani et Bagna, 2007; Ajeigbe et al., 2010 ; Boukar et al., 2016).

Selon Ahmed et al., (2009), 80 à 100\% de la production est perdue en raison des attaques des insectes. Les pucerons (Aphis craccivora Koch), les thrips des fleurs (Megalurothrips sjostedti Tryb.), les foreurs de gousses (Maruca vitrata Fabricius) et les punaises brunes Clavigralla tomentosicollis Stal) affectent la culture, de sa mise en place jusqu'à sa récolte (Madamba, 2006 ; Egho, 2010 ; Issoufou et al., 2017). La pyrale foreuse des fleurs et de gousses, $M$. vitrata est un important ravageur pouvant entraîner des pertes de l'ordre de 20 à $80 \%$ du rendement (Oyewale et Bamaiyi, 2013; Maina et al., 2014).

Plusieurs méthodes sont employées dans la lutte contre les insectes ravageurs du niébé parmi lesquelles s'inscrit l'utilisation des pesticides chimiques qui a toujours été privilégiée (Naseri et al., 2009). Cependant le risque élevé de contamination des personnes, des animaux et de l'environnement lié à l'emploi des produits chimiques a motivé le développement des programmes de recherche sur les méthodes alternatives répondants aux exigences d'ordre économique, écologique et toxicologique (Mukendi, 2013). Les biopesticides occupent une place de choix (Munyuli, 2009a; 2009b; 2009c). En Afrique sahélienne, les insecticides à base de dérivés du neem (Azadirachta indica) constituent une solution prometteuse et durable de lutte contre 
les ravageurs du niébé ( $\mathrm{Ba}$ et al., 2008 ; Biswas, 2013 ; Rabe et al., 2017a).

L'objectif de cette étude est d'identifier les principaux insectes ravageurs du niébé et les différentes méthodes de lutte utilisées dans la perspective de contribuer à la mise en place de programme de gestion durable de la culture.

\section{MATERIEL ET METHODES}

L'étude a été conduite du 07 au 22 septembre 2016 dans 9 villages de la région de Maradi de coordonnées géographiques $\left(13^{\circ} 30^{\prime} 00^{\prime \prime} \mathrm{N}\right.$ et $\left.07^{\circ} 06^{\prime} 06^{\prime \prime} \mathrm{E}\right)$ et 10 villages de

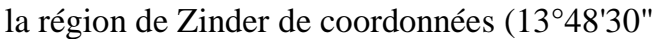
$\mathrm{N}$ et $\left.08^{\circ} 59^{\prime} 00^{\prime \prime} \mathrm{E}\right)$. Le choix des villages a été fait à partir d'une liste préalablement établie par les organisations des producteurs partenaires. Un focus groupe regroupant des personnes de sexe et de tranche d'âge différents a été utilisé pour collecter les données. Le questionnaire utilisé comporte les points suivants :

- L'activité socioéconomique des producteurs : les proportions de personnes pratiquant l'agriculture, l'élevage et autres occupations ;

- Les superficies des terres cultivées et le rendement de la culture : des moyennes ont été calculées par village à partir des chiffres avancés par au moins dix chefs d'exploitation ;

- Les contraintes de la culture du niébé : par village, les producteurs après un consensus ont énuméré les contraintes principales et secondaires de la culture du niébé ;

- Les principaux insectes ravageurs des cultures : les groupes des répondants ont listé les ravageurs primaires, occasionnant régulièrement des pertes à la culture du niébé et les ravageurs secondaires non moins importants, mais se manifestant occasionnellement sur la légumineuse ;

- La connaissance des producteurs sur les ravageurs : les réponses des groupes par village sur les organes attaqués, la nature des dégâts des pucerons, de la punaise brune et de $M$. vitrata ont été évaluées. Les niveaux de pertes ont été appréciés par village et par ravageur selon une grille à trois niveaux (faibles, moyens et élevés).

- $\quad$ Les méthodes de lutte contre les insectes ravageurs : pour chacun des villages, les groupes de producteurs ont listé les principales méthodes utilisées pour la gestion des ravageurs du niébé.

\section{Analyses statistiques}

Le test de student a été utilisé pour comparer les superficies des champs exploités et la production du niébé selon les régions. Le test de khi-carré a été effectué pour comparer les proportions des différentes réponses relatives aux variétés de niébé cultivées, les types d'associations de cultures, les contraintes de la culture du niébé, la connaissance des producteurs sur les insectes ravageurs et les méthodes de lutte employées pour le contrôle des ravageurs selon les régions. Le seuil de significativité est de 5\%. L'analyse a été faite avec le logiciel SPSS (IBM SPSS Statistics) version 20.

\section{RESULTATS}

L'étude a concerné 557 personnes dont $17 \%$ de femmes. L'âge des répondants a varié de 16 à 60 ans et leur activité principale est l'agriculture. L'acquisition des champs se fait dans la plupart des villages par héritage. Toutefois $11,1 \%$ des personnes enquêtées à Maradi affirment avoir effectué des opérations d'achat des champs.

La superficie totale des champs exploités est en moyenne de 2,7 ha/famille pour les deux régions avec des superficies utiles 1,4 fois plus importantes dans la région de Zinder par rapport à Maradi. La surface moyenne attribuée à la culture du niébé est de $1,04 \mathrm{ha} /$ famille et ces terres sont 1,9 fois plus importantes dans la région de Zinder comparée à celle de Maradi. Les rendements selon les producteurs sont en moyenne de 119 $\mathrm{kg} / \mathrm{ha}$ dans la région de Zinder et $138 \mathrm{~kg} / \mathrm{ha}$ à Maradi.

Pour $52,2 \%$ des villages enquêtés, les variétés utilisées dans les deux régions sont 
les locales (Dan illa, Oloka, SA-baba-Sata) suivies des variétés améliorées dont la IT90K37-1-2. Les associations de cultures de types mil-sorgho-niébé, sorgho-niébé, milsorgho-niébé-arachide et mil-niébé sont les plus rencontrées dans les deux régions (Tableau 1). Cependant l'association milniébé est la plus pratiquée et concerne 47,2\% des villages enquêtés.

Deux principales contraintes ont été rapportées au niveau des villages (Tableau 2). Les proportions de réponses sont comparables entre les deux régions. La pression des insectes ravageurs a été mentionnée par plus de $2 / 3$ des villages et celle relative à la pauvreté des sols par moins d'un tiers des villages. Ces deux contraintes sont les problèmes récurrents qui affectent négativement la production du niébé. L'insuffisance des pluies et les infestations par la plante parasite Striga gesnerioides (Willd.) sont les contraintes secondaires mentionnées respectivement par 57,2 et 42,8 $\%$ des villages au niveau des deux régions.

Comme contraintes entomologiques, deux ravageurs primaires ont été mentionnés et les proportions de réponses sont comparables entre les deux régions (Tableau 3). Il s'agit du puceron A. craccivora rapporté par plus de $2 / 3$ des villages et de la punaise brune $C$. tomentosicollis qui a été évoquée par moins d'un tiers des villages. Cette dernière se reproduit sur les plants de niébé causant le rabougrissement des graines et la déformation des gousses.

Pour les ravageurs secondaires, la foreuse de gousses $M$. vitrata a été rapportée par une proportion de villages environs trois fois plus importante dans la région de Maradi comparativement à celle de Zinder. Selon les producteurs, ses attaques sont plus importantes au cours des années ou les pluies sont régulières. Le coléoptère Mylabris sp. a été évoqué par moins de $30 \%$ des villages des deux régions. Le Criquet sénégalais Oeudaleus senegalensis Krauss a été uniquement rapporté dans la région de Zinder. Des pertes importantes sont souvent notées sur les plants du niébé en début de saison et en phase de fructification.

Pour la connaissance des pucerons, les réponses ont été comparables au niveau des deux régions (Tableau 4). A. craccivora s'attaque à la plante entière et cause le rabougrissement des plants. Les bonnes réponses ont été données par plus de $68 \%$ des villages. Son impact négatif sur le rendement a été jugé «moyen » à «élevé » par la totalité des villages enquêtés.

Pour la punaise brune $C$. tomentosicollis, par rapport aux organes de la plante attaqués et aux signes des dégâts, la totalité des villages a donné de bonnes réponses (Tableau 5). Les pertes sur la légumineuse ont été jugées élevées par plus de $75 \%$ des villages des deux régions.

Dans le cas de M. vitrata, les proportions de réponses ont été comparables entre les régions (Tableau 6). Le ravageur a été décrit comme s'attaquant uniquement aux gousses de la légumineuse par 59,5\% des villages alors qu'il infeste aussi les fleurs. Pour la nature des dégâts, $76,2 \%$ des villages ont mentionné la perforation des gousses, alors que l'espèce est aussi responsable de la chute des fleurs. Pour l'incidence sur la production, plus de $90 \%$ des producteurs l'ont jugée «moyenne » à «élevée »

En ce qui concerne les méthodes de lutte contre les insectes ravageurs, au niveau des deux régions, plus d'un tiers des villages ne fait rien, moins d'un tiers d'entre eux utilise des produits chimiques et moins d'un sixième fait recours aux biopesticides (Tableau 7). La destruction manuelle des insectes en champ a été évoquée au niveau de 3 villages de la région de Zinder.

Les biopesticides sont connus par 79\% des villages des deux régions. Boscia senegalensis (Pers.) Lam, le piment Capsicum sp. et le Neem sont les espèces végétales citées. Le biopesticide à base de grains de neem est connu par 53\% des villages. Son mode de préparation et d'utilisation a été mentionné au niveau de 4 villages des deux régions. 
Tableau 1 : Proportion des réponses sur les types d'associations de culture pratiquées au niveau des régions de Maradi et Zinder.

\begin{tabular}{lllllll}
\hline Questions & Réponses & Maradi & Zinder & Moyenne & $\mathbf{X}^{\mathbf{2}(2 d . f)}$ & Signification \\
\hline \multirow{2}{*}{ Type } & Mil-niébé & $44,4 \%$ & $50,0 \%$ & $47,2 \%$ & 0,05 & $*$ \\
d'association & Sorgho-niébé & $22,2 \%$ & $10,0 \%$ & $16,1 \%$ & 0,53 & $\mathrm{~ns}$ \\
& Mil-sorgho-niébé & $22,2 \%$ & $10,0 \%$ & $16,1 \%$ & 0,53 & $\mathrm{~ns}$ \\
& Mil-sorgho-arachide-niébé & $11,1 \%$ & $30,0 \%$ & $20,6 \%$ & 1,01 & $\mathrm{~ns}$ \\
\hline
\end{tabular}

*p=0,05; ns: différence non significative.

Tableau 2: Proportion des réponses des enquêtés sur les principales contraintes de la culture du niébé dans les régions de Maradi et Zinder.

\begin{tabular}{|c|c|c|c|c|c|c|}
\hline Contraintes & & Maradi & Zinder & Moyenne & $\mathrm{X}^{2}(2 \mathrm{~d} . \mathrm{f})$ & Signification \\
\hline Principales & Insectes ravageurs & $66,7 \%$ & $70,0 \%$ & $68,4 \%$ & 0.02 & ns \\
\hline contraintes & Pauvreté des sols & $33,3 \%$ & $30,0 \%$ & $31,6 \%$ & 0.02 & ns \\
\hline Contraintes & Insuffisance & $44,4 \%$ & $70,0 \%$ & $57,2 \%$ & 1.26 & ns \\
\hline \multirow[t]{2}{*}{ Secondaires } & pluies & & & & & \\
\hline & Parasite Striga & $55,6 \%$ & $30,0 \%$ & $42,8 \%$ & 3.45 & $\mathrm{~ns}$ \\
\hline
\end{tabular}

ns: différence non significative.

Tableau 3: Fréquence des réponses sur les principaux insectes ravageurs de la culture du niébé mentionnés par les enquêtés au niveau des régions de Maradi et Zinder.

\begin{tabular}{|c|c|c|c|c|c|c|}
\hline \multicolumn{2}{|c|}{ Insectes ravageurs } & \multirow{2}{*}{$\begin{array}{l}\text { Maradi } \\
55,6 \%\end{array}$} & \multirow{2}{*}{$\begin{array}{l}\text { Zinder } \\
80,0 \%\end{array}$} & \multirow{2}{*}{$\begin{array}{l}\text { Moyenne } \\
67,8 \%\end{array}$} & \multirow{2}{*}{$\frac{\mathbf{X}^{\mathbf{2}(2 \mathbf{d} . \mathbf{f})}}{1,31}$} & \multirow{2}{*}{$\begin{array}{l}\text { Signification } \\
\mathrm{ns}\end{array}$} \\
\hline Ravageurs primaires & Pucerons & & & & & \\
\hline & Punaises & $44,4 \%$ & $20,0 \%$ & $32,2 \%$ & 1.31 & ns \\
\hline \multirow[t]{3}{*}{ Ravageurs secondaires } & M. vitrata & $88,9 \%$ & $30,0 \%$ & $59,4 \%$ & 6,73 & ns \\
\hline & Mylabres & $11,1 \%$ & $40,0 \%$ & $25,5 \%$ & 2,039 & $\mathrm{~ns}$ \\
\hline & Criquets & & $30,0 \%$ & $15,0 \%$ & - & - \\
\hline
\end{tabular}

ns: différence non significative.

Tableau 4: Proportion des réponses des producteurs sur les parties de la plante infestées et l'incidence des pucerons sur la culture du niébé dans les régions de Maradi et Zinder.

\begin{tabular}{|c|c|c|c|c|c|c|}
\hline Paramètres & Réponses & Zinder & Maradi & Moyenne & $\mathrm{X}^{2}(2 \mathrm{~d} . \mathrm{f})$ & Signification \\
\hline $\begin{array}{lll}\text { Partie } & \text { de } & \text { la }\end{array}$ & Feuille & $11,1 \%$ & - & $5,6 \%$ & 1,06 & ns \\
\hline plante & Fleurs & $11,1 \%$ & $11,1 \%$ & $11,1 \%$ & 0,00 & ns \\
\hline attaquée & Plante entière & $77,8 \%$ & $88,9 \%$ & $83,3 \%$ & 0,40 & ns \\
\hline \multirow[t]{2}{*}{$\begin{array}{l}\text { Nature } \\
\text { dégâts }\end{array}$} & $\begin{array}{l}\text { Rabougrissement } \\
\text { de la plante }\end{array}$ & $66,7 \%$ & $77,8 \%$ & $72,2 \%$ & 0,27 & $\mathrm{~ns}$ \\
\hline & Assèchement de & $33,3 \%$ & $22,2 \%$ & $27,8 \%$ & 0,27 & ns \\
\hline
\end{tabular}




\begin{tabular}{lllllll}
\hline & la plante & & & & & \\
Evaluation des & Faibles & - & - & - & - & \\
\multirow{2}{*}{ pertes } & Moyennes & $33,3 \%$ & $66,7 \%$ & $50,0 \%$ & 2,00 & $\mathrm{~ns}$ \\
& Elevées & $66,7 \%$ & $33,3 \%$ & $50,0 \%$ & 2,00 & $\mathrm{~ns}$ \\
\hline ns: différence non significative & &
\end{tabular}

ns : différence non significative.

Tableau 5 : Proportion des réponses des enquêtés sur les parties de la plante attaquées et l'incidence des punaises brunes sur la culture du niébé dans les régions de Maradi et Zinder.

\begin{tabular}{lllllll}
\hline Paramètres & Réponses & Zinder & Maradi & Moyenne & $\mathbf{X}^{2}$ (2d.f) & signification \\
\hline Partie de la plante & Fleurs & $0 \%$ & $0 \%$ & $\%$ & - & - \\
attaquée & Gousses & $100,0 \%$ & $100,0 \%$ & $100,0 \%$ & - & - \\
& Fleurs et gousses & - & - & - & - & - \\
\multirow{2}{*}{ Nature des dégâtsts } & Rabougrissement & $100,0 \%$ & $100,0 \%$ & $100,0 \%$ & - & - \\
& des gousses & & & & & \\
\multirow{2}{*}{$\begin{array}{l}\text { Estimation } \\
\text { pertes }\end{array}$} & Faibles & - & $11,1 \%$ & $5,50 \%$ & 0,48 & $\mathrm{~ns}$ \\
& Moyennes & $25,00 \%$ & $11,1 \%$ & $18,0 \%$ & 0,41 & $\mathrm{~ns}$ \\
& Elevées & $75,00 \%$ & $77,8 \%$ & $76,4 \%$ & 0,01 & $\mathrm{~ns}$ \\
\hline
\end{tabular}

ns: différence non significative.

Tableau 6 : Proportion des réponses des producteurs sur les parties de la plante infestées et l'incidence de $M$. vitrata sur la culture du niébé dans les régions de Maradi et Zinder.

\begin{tabular}{lllllll}
\hline Paramètres & Réponses & Zinder & Maradi & Moyenne & $\mathbf{X}^{\mathbf{2}(2 d . f)}$ & signification \\
\hline Partie de la & Fleurs & $33,3 \%$ & - & $16,6 \%$ & 2,59 & $\mathrm{~ns}$ \\
plante & Gousses & $33,3 \%$ & $85,7 \%$ & $59.5 \%$ & 5,83 & $\mathrm{~ns}$ \\
attaquée & Fleurs et gousses & $33,3 \%$ & $14,3 \%$ & $23,8 \%$ & 2,59 & $\mathrm{~ns}$ \\
\multirow{2}{*}{ Nature des } & Perforation des gousses & $66,7 \%$ & $85,7 \%$ & $76,2 \%$ & 0,02 & $\mathrm{~ns}$ \\
dégâts & Perte des fleurs et & $33,3 \%$ & $14,3 \%$ & $23,8 \%$ & 0,02 & $\mathrm{~ns}$ \\
& perforation des gousses & & & & & \\
Estimation & Faibles & & $14,3 \%$ & $7.1 \%$ & 0,48 & $\mathrm{~ns}$ \\
des pertes & Moyennes & $33,3 \%$ & $57,1 \%$ & $45,2 \%$ & 0,48 & $\mathrm{~ns}$ \\
& Elevées & $66,7 \%$ & $28,6 \%$ & $47,6 \%$ & 1,27 & $\mathrm{~ns}$ \\
\hline
\end{tabular}

ns: différence non significative. 
Tableau 7 : Proportion des réponses des enquêtés sur les méthodes de lutte employées contre les insectes ravageurs dans les régions de Maradi et Zinder.

\begin{tabular}{llcclll}
\hline Questions & Réponses & Maradi & Zinder & Moyenne & $\mathbf{X}^{\mathbf{2}(2 d . f)}$ & signification \\
\hline \multirow{3}{*}{ Méthodes de lutte } & Rien & $44,5 \%$ & $30,0 \%$ & $37,25 \%$ & 0,42 & $\mathrm{~ns}$ \\
& Pesticides & $44,4 \%$ & $20,0 \%$ & $32,2 \%$ & 1,31 & $\mathrm{~ns}$ \\
& Biopesticides & $11,1 \%$ & $20,0 \%$ & $15,55 \%$ & 0,28 & $\mathrm{~ns}$ \\
& Mécanique & - & $30,0 \%$ & $15,0 \%$ & 3,22 & $\mathrm{~ns}$ \\
\hline
\end{tabular}

ns: différence non significative.

\section{DISCUSSION}

Les données collectées ont permis de caractériser les exploitations agricoles au niveau des deux zones d'étude. Les familles disposent de champs de petites tailles de 2 à 3 ha pour la culture des céréales et des légumineuses, le tiers des surfaces est consacré à la culture du niébé. Cette légumineuse est souvent associée au mil qui est l'alimentation de base des familles. Le niébé est aussi reconnu pour ses multiples avantages dans l'alimentation des ménages, du bétail mais aussi contribue à l'amélioration des systèmes de cultures et de la fertilité des sols (Tarawali et al., 2002). De par leurs composantes et leur mode de fonctionnement, ces exploitations des régions de Maradi et Zinder peuvent être considérées comme de type familial, car répondant aux critères définis par certains auteurs (Andrianantoandro et Bélières, 2015 ; Sourisseau et Even, 2015 ; Lacombe, 2016).

Les focus groupes ont fait ressortir principalement quatre contraintes de production. La pression des insectes ravageurs a été notifiée par plus de deux tiers des villages. Cette donnée corrobore les observations de plusieurs auteurs qui ont rapportés la sensibilité de cette légumineuse aux ravageurs (Dugjé et al., 2009 ; N'gbesso et al., 2013; Sreelakshmi et al., 2016 ; Issoufou et al., 2017 ; Oladejo et al., 2017 ; Rabe et al., 2017 ). Les producteurs au niveau des villages ont ressorti les deux ravageurs majeurs du niébé suivants :
Le puceron A. craccivora : c'est une des espèces la plus crainte et évoquée par plus des deux tiers des villages. Il s'agit d'un ravageur qui attaque le niébé à tous ses stades de développement. L'espèce est très prolifique, et les larves et adultes se nourrissent continuellement de la sève sur les plants de niébé (Choudhary et al., 2017). L'espèce est aussi vectrice des maladies virales (Angelella et al., 2018 ; van Leur et al., 2019). Les pertes occasionnées à la culture du niébé sont estimées entre 20 et $40 \%$ (Reddy et al., 2014).

La punaise brune $C$. tomentosicollis : elle infeste le niébé au cours de sa phase de fructification et développe au moins trois générations. Une femelle pond en moyenne 120 œufs. Les larves et les adultes vivent sur les plants se nourrissant continuellement sur les gousses et occasionnant des pertes de rendement de 17,5 à 26,5\% (Abdourahmane et al., 2018). En cas d'attaque sévère, les pertes de production peuvent atteindre $70 \%$ (Ujagir et Byrne, 2009).

Ces bioagresseurs sont bien connus des producteurs puisque $60 \%$ à $100 \%$ des villages ont décrit les organes de la plante attaqués et les signes de présence des ravageurs. Ils ont aussi jugé leurs impacts négatifs sur la production comme «moyen» et «élevé », ce qui est comparable aux niveaux de pertes mentionnés par les auteurs cités plus haut.

Les producteurs ont aussi listé les deux ravageurs suivants comme secondaires : 
M. vitrata: Il s'agit d'une pyrale connue comme ravageur primaire de plusieurs légumineuses. En zone sahélienne, l'espèce migre de la zone sud en saison pluvieuse pour se reproduire sur le niébé (Ba et al., 2009). M. vitrata se développe dans les bourgeons, les fleurs et les gousses (Dugjé et al., 2009). Selon Sharma (2004), les infestations sont plus importantes dans les inflorescences comparativement aux gousses. Les pertes de production associées à cette pyrale varient de 20 à $80 \%$ selon les années (Kanhere et al., 2012; Oyewale et Bamaiyi, 2013; Souna, 2018).

Mylabris sp.: Il s'agit de coléoptères qui envahissent la culture au stade floraison. Ils se nourrissent du pollen, des fleurs et souvent des gousses (Ajao et al. 2016). Une densité de 3 adultes par dix plants de niébé est requise pour noter une incidence économique sur la production du niébé (Durairaj et Ganapathy, 2000). Sur le Mungobean Vigna radiata L. Wilzeck, les pertes dues à ce ravageur varient de 11 à $90 \%$ selon les densités du ravageur (Dhavan et al., 2014).

Mylabris sp. a été évoqué par $25,5 \%$ des villages, mais l'insecte est bien visible dans les champs de niébé se nourrissant des fleurs en raison de sa coloration prononcée et sa grande taille.

Les données de cette étude, démontrent que le lépidoptère $M$. vitrata est méconnu des producteurs. Ils l'ont décrit comme ravageur des gousses, mais l'insecte se nourrit aussi des jeunes feuilles et des fleurs et est donc aussi responsable de la perte des fleurs. Ces résultats sont contraires à ceux rapportés au Ghana et au Bénin ou suite à une étude, 61 à $80 \%$ des répondants ont bien décrit l'insecte (Downham, 2005). Ces pays étant situés dans la partie sud de l'Afrique de l'ouest, sont marqués par un climat plus humide, propice au développement du ravageur, d'où il constitue un ravageur important du niébé (Sokame et al., 2015; Salifu and Yeboah, 2019). L'espèce pourrait être aussi rare dans les régions de Maradi et Zinder. Une étude conduite au Burkina Faso a déjà mis en évidence les possibilités de migration saisonnière du ravageur dans la partie centrale du pays en saison pluvieuse à partir de la zone sud sahélienne (Ba et al., 2009). Très peu de données sur le ravageur sont aussi disponibles au Niger. Des études sont donc à conduire pour déterminer le cycle de développement de cette pyrale et son incidence sur les rendements du niébé au niveau des latitudes des régions de Maradi et Zinder.

Les Thrips des fleurs M. sjöstedti n'ont pas été évoqués par les répondants des deux régions. Il s'agit d'un ravageur très important. Les larves et les adultes sont responsables de la chute des fleurs et peuvent causer des pertes de rendement de 20 à $100 \%$ (Ngakou et al., 2008; Agbahoungba et al. 2017).

Le criquet sénégalais $O$. senegalensis est un insecte polyphage qui s'attaque à toutes les cultures en zone sahélienne. Sa dynamique de population est liée à la pluviométrie (Maiga, 2008). En cas de fortes infestations, les pertes peuvent être considérables (Maiga, 2008). L'espèce est donc présente dans toutes les zones agricoles du Niger alors que dans la présente étude elle a été uniquement mentionnée dans la région de Zinder.

Pour les méthodes de lutte, seulement $32,2 \%$ utilisent les pesticides chimiques malgré leur impact négatif sur l'environnement et la santé humaine. Plus du tiers des villages ne fait rien comme déjà mentionné par Rabé et al. (2017b) malgré la pression importante des insectes ravageurs. Cette situation explique les faibles rendements du niébé de 129 et $138 \mathrm{~kg} / \mathrm{ha}$ qui sont en dessous des moyennes régionales (MA, 2018). Au Niger, il y a donc une faible utilisation des technologies de lutte phytosanitaire contrairement au Bénin, où $62 \%$ des producteurs utilisent pesticides chimiques et $26 \%$ emploient les produits botaniques (Downham, 2005).

Toutefois l'enquête a révélé que 53\% des villages connaissent bien les vertus du neem comme insecticide mais seulement $29,8 \%$ connaissent son mode préparatoire. 
Rabé et al. (2017b) a rapporté dans une étude effectuée dans la région de Maradi que seulement $7,4 \%$ des producteurs utilisent les extraits du neem. Cette technologie écologique et efficace est très peu valorisée par les producteurs des deux régions.

En plus des insectes ravageurs, les producteurs ont aussi évoqué d'autres contraintes non moins importantes. Il s'agit de la pauvreté des sols, l'insuffisance des pluies et le $S$. gesnesrioides. La pauvreté des sols est une contrainte considérée comme généralisée en raison de la surexploitation des terres. Des options pour la conservation du sol utilisant diverses techniques de lutte contre l'érosion et aussi des apports de la fumure organique sont développées (Kpadonou et al., 2017 ; Suzuki, 2017 ; Bado et Bationo, 2018).

Pour l'insuffisance des pluies et la pression de S. gesnerioides, des nouvelles variétés de niébé à résistance multiples sont disponibles (Yusuf et Zakari, 2016 ; Omoigui et al., 2017 ; Harou et al., 2018).

\section{Conclusion}

Il ressort de cette étude que le niébé est une composante importante de l'agriculture familiale nigérienne. Son rendement est bas en raison des pressions biotiques et des contraintes d'ordre édaphiques. La gestion des insectes ravageurs tels que la punaise brune, les pucerons, et la foreuse des gousses M.vitrata doit d'abord débuter par une sensibilisation des producteurs sur le diagnostic précoce des infestations et les étapes de leur développement sur la culture. Le taux d'utilisation des technologies de protection phytosanitaire devra être amélioré. Les pesticides chimiques utilisés par 32,2\% des villages sont dangereux pour la santé des utilisateurs, les consommateurs et l'environnement. La promotion de l'utilisation des dérivés du neem pourra aider les producteurs à réduire les pertes par les ravageurs et augmenter les rendements. Ainsi des travaux de recherche sont à mener pour mieux préciser les formulations, les doses et les fréquences de traitements du niébé en tenant compte de la pression des ravageurs dans les deux régions. Aussi une recherche d'accompagnement des producteurs semble nécessaire dans le contexte des deux régions, pour préciser l'incidence sur le rendement de certains ravageurs tels que la pyrale du niébé. Compte tenu aussi du temps de travail important que requiert la collecte, la préparation et l'application des biopesticides à base de neem, des possibilités de mise en place d'entreprises communautaires pour la production des formulations prêtes à l'emploi sont à explorer. Des efforts sont également à faire à travers des programmes de vulgarisation des technologies pour une agriculture de conservation et aussi l'utilisation des variétés améliorées à résistance multiples selon le contexte. $\mathrm{La}$ vulgarisation de ces technologies pourra se faire en utilisant le concept participatif de champ école paysan.

\section{CONFLIT D'INTERETS}

Aucun conflit d'intérêts potentiel n'a été signalé par les auteurs.

\section{CONTRIBUTIONS DES AUTEURS}

OAZ a élaboré le questionnaire d'enquête, collecté et analysé les données et rédigé l'article. IB a supervisé l'étude et la rédaction scientifique. $\mathrm{LA}$, MT et $\mathrm{BP}$ ont contribué à la correction du manuscrit.

\section{REMERCIEMENTS}

Les remerciements vont également à l'égard de tous ceux qui ont contribués à la collecte de données de terrain et aussi à la correction de cet article.

\section{REFERENCES}

Abdourahamane HM, Ibrahim B, Tamo M, Laouali A, Saadou M, Barry P. 2018. Etude des paramètres de reproduction et de développement de Clavigralla tomentosicollis Stål, 1855 (Hemiptera : Coreidae) et son incidence sur le rendement du niébé dans la région de Maradi au Niger. REV. RAMRES, 
Science de la Vie, de la Terre et Agronomie, 6(1): 42- 48.

Agbahoungba S, Karungi J, Odong TL, Badji A, Sadik K, Rubaihayo PR. 2017. Stability and extent of resistance of cowpea lines to flower bud thrips in Uganda. African Crop Science Journal, 25(1): 1-24. DOI : http://dx.doi.org/10.4314/acsj.v25i1.1

Ahmed BI, Onu I, Mudi L. 2009. Field bioefficacy of plant extracts for the control of post flowering insect pests of cowpea (Vigna unguiculata (L.) Walp.) in Nigeria. Journal of Biopesticides, 2(1): 37-43. http://agris.fao.org/agrissearch/search.do?recordID=IN20110000 22

Ajao FO, Osipitan AA, Pitan OR, Lawal OI. 2016. Effect of Plant Spacing on Abundance of Major Insect Pests of Cowpea [Vigna unguiculata (L.) Walp] and Crop Yield. Journal of Organic Agriculture and Environment, 4(1): 2433. DOI: $10.5923 /$ j.ijaf.20140401.04

Ajeigbe HA, Ekeleme F, Chikoye D. 2010. Improved Crop- livestock System for Enhanced Food Security and Income Generation in West Africa. Final Project Report, Gatsby Improved Crop-livestock Project (Project Number: GAT2833), International Institute of Tropical Agriculture (IITA), Ibadan, Nigeria, p50. Andrianantoandro VT, Bélières JF. 2015. L'agriculture familiale malgache entre survie et développement: organisation des activités, diversification et différenciation des ménages agricoles de la région des Hautes Terres. Revue Tiers Monde, 1(1): 69-88. DOI: 10.3917/rtm.221.0069

Angelella G, Nalam V, Nachappa P, White J, Kaplan I. 2018. Endosymbionts Differentially Alter Exploratory Probing Behavior of a Nonpersistent Plant Virus Vector. Microbial Ecology, 76(2): 453458. DOI: $10.1007 / \mathrm{s} 00248-017-1133-5$
Ba NM, Dabire C, Drabo I, Sanon A, Tamo M. 2008. Combinaison de la résistance variétale et d'insecticides à base de neem pour contrôler les principaux insectes ravageurs du niébé dans la région centrale du Burkina Faso. IITA Journal Articles, $\quad 30(1) . \quad$ DOI : https://hdl.handle.net/10568/92269

Ba NM, Margam VM, Binso-Dabire CL, Sanon A, McNeil JN, Murdock LL, Pittendrigh BR. 2009. Seasonal and regional distribution of the cowpea pod borer Maruca vitrata (Lepidoptera: Crambidae) in Burkina Faso. International Journal of Tropical Insect Science, $\quad$ 29(3): $\quad 109-113$. DOI: https://doi.org/10.1017/S17427584 0999021X

Bado VB, Bationo A. 2018. Integrated management of soil fertility and land resources in Sub-Saharan Africa: involving local communities. Advances in Agronomy, 150: 1-33. DOI: http://dx.doi.org/10.1016/bs.agron.2018. 02.001

Biswas GC. 2013. Comparative effectiveness of Neem extracts and synthetic organic insecticide against mustard aphid. Bangladesh Journal of Agricultural Research, 38(2): 181-187. DOI: https://doi.org/10.3329/bjar.v38i2.15881

Boukar O, Fatokun CA, Huynh BL, Roberts PA, Close TJ. 2016. Genomic tools in cowpea breeding programs: status and perspectives. Frontiers in Plant Science, 757(7): $\quad 1-13 . \quad$ DOI: 10.3389/fpls.2016.00757

Choudhary AL, Hussain A, Samota RG, Nehra S. 2017. Effect of biotic and abiotic factors on the incidence of aphid, Aphis craccivora Koch on cowpea. Journal of Pharmacognosy and Phytochemistry, 6(4): 1587-1590.

Dhavan SP, Wadaskar RM, Patil SC. 2014. Assessment of Economic Threshold Level for Blister Beetles (Mylabris phalerata) on Greengram. Indian Journal of Ecology, 41(2): 304-306. 
Souna DA. 2018. Stratégies de réussite parasitaire du parasitoïde larvaire Therophilus javanus et de ses potentialités d'utilisation en lutte biologique contre Maruca vitrata le lépidoptère foreur de gousse de niébé Vigna unguiculata en Afrique de l'Ouest. Thèse de doctorat en Parasitologie, Université MontpellierUniversité d'Abomey-Calavi, Bénin.

Downham M. 2005. Implementing Pheromone traps and other new technologies for control of cowpea insect pests in West Africa through farmer field schools. Crop Protection Programme. http://www.fao.org/docs/eims/upload/agr otech/1975/R8300_FTR.pdf

Dugjé IY, Omoigui LO, Ekeleme F, Kamara AY, Ajeigbe H. 2009. Production du niébé en Afrique de l'Ouest : Guide du paysan. Document de l'IITA, p.20. Consulté le 18/06/2019.

Durairaj C, Ganapathy N. 2000. Yield losses caused by blister beetle, Mylabris sp. (Coleoptera: Meloidae) in pigeon pea and its economic injury level in cowpea. Tropical Agriculture, 77(2): 133-136.

Egho EO. 2010. Comparative studies on insect species of cowpea (Vigna unguiculata) (L) Walp in two agroecological zones during the early cropping season, in Delta State, southern Nigeria. Agriculture and Biology Journal of North America, (1): 946-949. DOI:10.5251/abjna.2010.1.5.946.949

FAO (Food and Agriculture Organization). 2014. FAOSTAT.

FAO. http://faostat3.fao.org/download/Q/QC/E $1 / 04 / 2014$

Food and Agriculture Organization Corporate Statistical Database (FAOSTAT) 2016. FAO.

WWW.fao.org/faostat/en/\#data/QC

Harou A, Hamidou F, Bakasso Y. 2018. Performances morpho-physiologiques et agronomiques du niébé [Vigna unguiculata (L.) Walpers] en conditions du déficit hydrique. Journal of Applied
Biosciences, 128 : 12874-12882. DOI : https://dx.doi.org/10.4314/jab.v128i1.1

Issoufou $\mathrm{OH}$, Boubacar $\mathrm{S}$, Adam T, Boubacar Y. 2017. Identification des insectes, parasites et évaluation économique de leurs pertes en graines sur les variétés améliorées et locale de niébé en milieu paysan à Karma (Niger). International Journal of Biological and Chemical Sciences, 11(2): 694-706. DOI: http://dx.doi.org/10.4314/ijbcs.v11i2.13

Kanhere RD, Patel VN, Umbarkar PS, Kakde AM. 2012. Bio-efficacy of different insecticides against spotted pod borer, Maruca testulalis (Geyer) infesting cowpea. Legume Research, (35): 44-46. DOI:

https://arccjournals.com/journal/legumeresearch-an-internationaljournal/ARCC503

Kpadonou RAB, Owiyo T, Barbier B, Denton F, Rutabingwa F, Kiema A. 2017. Advancing climate-smart-agriculture in developing drylands: Joint analysis of the adoption of multiple on-farm soil and water conservation technologies in West African Sahel. Journal of Land Use Policy, (61): 196-207. DOI: https://doi.org/10.1016/j.landusepol.2016 .10 .050

Lacombe P. 2016. L'agriculture familiale: rengaine ou nouveauté? Natures sciences sociétés, 24(2): 123-135. DOI : https://doi.org/10.1051/nss/2016021

MA. 2012. Rapport d'évaluation préliminaire des récoltes 2012, et résultats de la campagne agricole 2012/2013, République du Niger, Ministère de l'agriculture. Direction des Statistiques, République du Niger, $26 \mathrm{P}$. http://www.recaniger.org/spip.php?article575

MA. 2018. Rapport d'évaluation de la campagne agricole d'hivernage 2017 et perspectives alimentaires 2017-2018. Direction générale des ressources, direction des statistiques, $42 \mathrm{P}$. http://images.agriprofocus.nl/upload/post 
/Annuaire-Disponibilite

semences_Niger_20161460556868.pdf

Madamba R, Grubben GJH, Asante IK, Akromah R. 2006. Vigna unguiculata (L.) Walp. Plant Resources of Tropical Africa, 1: 221-229.

Maiga IH, Lecoq M, Kooyman C. 2008. Ecology and management of the Senegalese grasshopper Oedaleus senegalensis (Krauss 1877) (Orthoptera: Acrididae) in West Africa: review and prospects. Annales de la Société Entomologique de France (Taylor \& Francis Group), 44(3): 271-288. DOI: https://doi.org/10.1080/00379271.2008.1 0697563

Maina UM, Sastawa BM, Biu BM. 2014. Evaluation of cultivars and insecticides on insect pests and grain loss of rainfed cowpea Vigna unguiculata (L.) Walp.) at Baga, Lake Chad shore area of Nigeria. Journal of Entomology and Nematology, 6(11):161-168.

DOI: https://doi.org/10.5897/JEN2014. 0109

Mehinto JT, Atachi P, Elégbédé M, Kpindou OKD, Tamò M. 2014. Efficacité comparée des insecticides de natures différentes dans la gestion des insectes ravageurs du niébé au Centre du Bénin. Journal of Applied Biosciences, 84 (1): 7695-7706.

DOI: http://dx.doi.org/10.4314/jab.v84i1.1

Mukendi R, Tshlenge P, Kabwe C, Munyuli TMB. 2013. Efficacité des plantes médicinales dans la lutte contre Ootheca mutabilis sahlb. (chrysomelidae) en champ de niébé (vigna unguiculata (1.) Walp.) en RDC. Lebanese Science Journal, $\quad$ 15(1). DOI: http://lsj.cnrs.edu.lb/wpcontent/uploads/2015/12/munyuli.pdf

Munyuli TMB. 2009a. Is Pardosa pseudoannulata an effective predator agent of Aphis craccivora in Uganda and in Democratic Republic of Congo? Tunisian Journal of Plant Protection, 4(1): 91-98.
Munyuli TMB. 2009b. Effects of native insect predators on population densities of Aphis craccivora and yields of Vigna unguiculata and Arachis hypogeae grown under various cropping systems, in Kivu Province, Eastern Democratic Republic of Congo. Tunisian Journal of Plant Protection, 4(2): 197-209.

Munyuli TMB. 2009c. On-farm storages participatory evaluation and validation of the capability of native botanicals for control of bean bruchids (Acanthoscelides obtectus L., Coleoptera: Bruchidae) in South-Kivu province, eastern of Democratic Republic of Congo. Tropicultura, 27(3): 174-183.

DOI: http://www.tropicultura.org/text/v27n3/1 74

N'Gbesso FPM, Fondio L, Dibi BEK, Djidli HA, Kouame CN. 2013. Étude des composantes du rendement de six variétés améliorées de niébé [Vigna unguiculata (L.) Walp]. Journal of Applied Biosciences, 63: 4754-4762. DOI:

http://dx.doi.org/10.4314/jab.v63i1.8724 9

Naseri B, Fathipour Y, Talebi AA. 2009. Population density and spatial distribution pattern of Empoasca decipiens (Hemiptera: Cicadellidae) on different beans species. J. Agric. Technol., 11: 239-248.

Ngakou A, Tamò M, Parh IA, Nwaga D, Ntonifor NN, Korie S, Nebane CLN. 2008. Management of cowpea flower thrips, Megalurothrips Sjostedti (Thysanoptera: Thripidae), in Cameroon. Journal of Crop Protection, 27: 481-488. DOI: 10.1016/j.cropro.2007.08.002

Oladejo AS, Boukar O, Fatokun CA, Fatokun IO. 2017. Genetic analysis of thrips resistance in cowpea (Vigna unguiculata [L.] Walp.) Euphytica, 213(9): 213-216. DOI: http://dx.doi.org/10.1007/s10681017-2001-6 
Omoigui LO, Ajeigbe HA, Akinwale RO, Timko MP, Oyekunle M, Bello LL. 2017. Performance of cowpea varieties under Striga gesnerioides (Willd.) Vatke infestation using biplot analysis. Euphytica, 213(11): 244. DOI: http://dx.doi.org/10.1007/s10681-0172034-x

Oyewale RO, Bamaiyi LJ. 2013. Management of Cowpea Insect Pests. Scholars Academic Journal of Biosciences, 1(5): 217-226.

https://pdfs.semanticscholar.org/76bc/db 8ddbfd4a68813349011e77cf8f3e532c1a. pdf

Rabé MM, Baoua IB, Sitou L, Amadou L. 2017a. Champ école paysan, une approche participative pour l'amélioration du rendement du niébé: résultats d'expériences pilotes conduites dans les régions de Maradi et Zinder au Niger. Agronomie Africaine, 29(2):1-9. DOI :

https://www.ajol.info/index.php/aga/artic le/view/163178

Rabé MM, Ibrahim B, Razack A, Lawali S, Laouali A, Barry P, Saadou M. 2017b. Les déterminants socioéconomiques de l'adoption des technologies améliorées de production du niébé diffusées par les champs écoles paysans dans les régions de Maradi et Zinder au Niger. International Journal of Biological and Chemical Sciences, 11(2): 744-756. DOI:

https://dx.doi.org/10.4314/ijbcs.v11i2.17

Reddy DS, Latha MP, Chowdary LR, Kumar LR. 2014. Efficacy of chemical and botanical against cowpea aphid [Aphis craccivora (Koch)]. Bioinfolet, (11): 853-854.

Salifu B, Yeboah AAAS. 2019. Efficacy of spring onion (Allium fistulosum) leaf extract for controlling major field insect pests of cowpea (Vigna unguiculata L.) in the guinea savannah Agroecological zone of Ghana. Journal of Entomology and Zoology Studies, 7(1): 730-733.
Sani RM, Bagna A. 2007. Adoption of improved seed s of millet and cowpea by farmers in Madarounfa district, Niger republic. Continental Journal of Agricultural Economics, (1): 1-6.

Sharma HC. 2004. Legume pod borer, Maruca vitrata: Insect plant relationships. Insect Science and its Application, (25): 73-75.

Sokame BM, Tounou AK, Datinon B, Dannon EA, Agboton C, Srinivasan R, Tamo M. 2015. Combined activity of Maruca vitrata multi-nucleopolyhedrovirus, MaviMNPV, and oil from neem, Azadirachta indica Juss and Jatropha curcas L., for the control of cowpea pests. J. Crop Protection, (72): 150-157. DOI :

https://doi.org/10.1016/j.cropro.2015.03. 016

Sourisseau JM, Even MA. 2015. Transformations agricoles et agricultures familiales : quelques défis mis en lumière durant l'Année internationale de l'agriculture familiale. Cahiers d'Agriculture, (24): 201-203. DOI : 10.1684/agr.2015.0764

Sreelakshmi P, Paul A, Beevi NS, Sheela MS, Kumar PN. 2016. Management of resistant populations of legume pod borer, Maruca vitrata (Fab.) (Lepidoptera: Crambidae) using new generation insecticides. Journal of Environment and Ecology, 34(3): 917921.

Stoilova T, Pereira G. 2013. Assessment of the genetic diversity in a germplasm collection of cowpea (Vigna unguiculata (L.) Walp.) using morphological traits. African Journal of Agricultural Research, 8(2): 208-215. DOI: 10.5897/AJAR12.1633

Suzuki K, Matsunaga R, Hayashi K, Matsumoto N, Tobita S, Bationo A, Okada K. 2017. Effects of long-term application of mineral and organic fertilizers on dynamics of nitrogen pools in the sandy soil of the Sahel region, 
Niger. Agriculture, Ecosystems \& Environment, (242): 76-88. DOI: http://doi.org/10.1016/j.agee.2017. 03.004

Tarawali SA, Singh BB, Gupta SC, Tabo R, Harris F, Nokoe S, Fernandez-Rivera S, Bationo A, Manyong VM, Makinde K, Odion EC. 2002. Cowpea as a key factor for a new approach to integrated croplivestock systems research in the dry savannas of West Africa. In Challenges and Opportunities for Enhancing Sustainable Cowpea Production, Fatokun CA, Singh BB, Kormawa PM, Tamò $\mathrm{M}$ (eds). International Institute of Tropical Agriculture (IITA): Ibadan, Nigeria; 233-251. https://hdl.handle.net/10568/49692

Ujagir R, Byrne O. 2009. Insect pests and their management. In The Lentil: Botany,
Production and Uses, Erskine W, Muehlbaue FJ, Sarker A, Sharma B, (eds). Wallingford: CABI; 282-305.

Van Leur J, Duric Z, George J, Boschma S. 2019. Alfalfa mosaic virus infects the tropical legume Desmanthus virgatus in Australia and the potential role of the cowpea aphid (Aphis craccivora) as the virus vector. Australasian Plant Disease Notes, $\quad$ 14(1): $\quad 3 . \quad$ DOI : https://link.springer.com/article/10.1007/ s13314-019-0335-8

Yusuf AU, Zakari FS. 2016. Influence Of Plant Spacing on Insect Pest Infestation and Yield Of Some Cowpea (Vigna unguiculata L. Walp.) Varieties in Kano, Nigeria. Journal of Organic Agriculture and Environment, 4(1). DOI : http://oaptin.org/index.php/JOAEN/articl e/view/13. 